\title{
GCU
}

Glasgow Caledonian

University

University for the Common Good

\section{A semantic web-based approach for generating parametric models using RDF}

Sadeghineko, Farhad; Kumar, Bimal; Chan, Warren

Published in:

Advanced Computing Strategies for Engineering

DOI:

10.1007/978-3-319-91638-5_20

Publication date:

2018

Document Version

Author accepted manuscript

Link to publication in ResearchOnline

Citation for published version (Harvard):

Sadeghineko, F, Kumar, B \& Chan, W 2018, A semantic web-based approach for generating parametric models using RDF. in IFC Smith \& B Domer (eds), Advanced Computing Strategies for Engineering: 25th EG-ICE International Workshop 2018. Lecture Notes in Computer Science, vol. 10864, Springer, Switzerland, pp. 361377, 25th EG-ICE International Workshop, Lausanne, Switzerland, 11/06/18. https://doi.org/10.1007/978-3-31991638-5_20

\section{General rights}

Copyright and moral rights for the publications made accessible in the public portal are retained by the authors and/or other copyright owners and it is a condition of accessing publications that users recognise and abide by the legal requirements associated with these rights.

Take down policy

If you believe that this document breaches copyright please view our takedown policy at https://edshare.gcu.ac.uk/id/eprint/5179 for details of how to contact us. 


\title{
A Semantic Web-based Approach for generating parametric models using RDF
}

\author{
Farhad Sadeghineko ${ }^{1}$, Bimal Kumar ${ }^{1}$, and Warren Chan $^{2}$ \\ 1 Glasgow Caledonian University, Construction and Surveying Department, \\ Glasgow, UK, G4 0BA \\ \{farhad.sadeghineko, b.kumar\}@gcu.ac.uk \\ 2 Glasgow Caledonian University, Computer, Communications \& Interactive Systems, \\ Glasgow, UK, G4 0BA \\ warren.chan@gcu.ac.uk
}

\begin{abstract}
Semantic-rich 3D parametric models, like Building Information Models (BIMs) are becoming the main information source during the entire lifespan of an asset. The use of BIM in existing buildings has been hampered by the challenges surrounding the limitations of existing technologies for developing retrofit models. Some progress has been recently made in generating non-parametric models from the Point Cloud Data (PCD). However, a proper fully developed parametric model is still some way away. In this paper, challenges are addressed by reviewing the stateof-the-art before presenting our approach. The aim of our approach is to apply the Semantic Web Technologies for generating parametric models using PCD as primary data. The Semantic Web as a set of standards and technologies is used for providing an appropriate framework for storing, sharing, and reusing the semantics of information on the web. Building elements are recognized in PCD, and the concept of Resource Description Framework (RDF) as a Semantic Web technology and a standard model for interchanging the data on the web is then used to markup detected elements. The RDF data is then standardized to Industry Foundation Classes (IFC) as an open standard building data model to generate the parametric model of the asset utilizing BIM software that supports IFC. Some parts of this ongoing research are performed manually, and the future work is to implement the process automatically. Primary results are quite promising and should be of interest to the modeling of all kinds of assets, in particular, Historical Building Information Modelling (HBIM).
\end{abstract}

Keywords: Building Information Modelling (BIM), Semantic Web, Resource Description Framework (RDF).

\section{Introduction}

Building Information Modelling (BIM) is utilized in Architecture, Engineering, and Construction (AEC), Facility Management (FM), and other domains to provide information concerning the building [1]. Semantic-rich three-dimensional 
parametric models, like Building Information Models (BIMs) are fast becoming the main source of information during the entire life-cycle of an asset $[2,3,4]$. The use of BIM impacts different aspects of an asset consisting in the precision of the planning phase, the decision-making process, the improvement of data management, the enhancement of the productivity, flexibility, energy efficiency, sustainability, and safety in construction $[2,5,6,7,8]$. Accurate BIMs improve the performance and facility management of an asset [9]. As a matter of fact, after the completion of construction, BIMs that are mapped from the as-designed condition of an asset do not necessarily match to the originally constructed building (as-is condition) due to the changes applied to the building during the construction process in different stages [10]. On the other hand, in some unique circumstances like existing assets and historical environments, the asset may not have a proper $3 \mathrm{D}$ model - the only available data are $2 \mathrm{D}$ drawings and corresponding documents. Hence several approaches have been proposed during the past years to make the process of capturing BIMs as effective and efficient as possible by developing automated or semi-automated approaches to map parametric models. The general workflow of capturing BIMs using 3D point measurements gathered from the asset can be classified into two directions including 'Scan and BIMs' and 'Scan to BIMs' (Fig.1) [11,12]. In the case of the availability of the

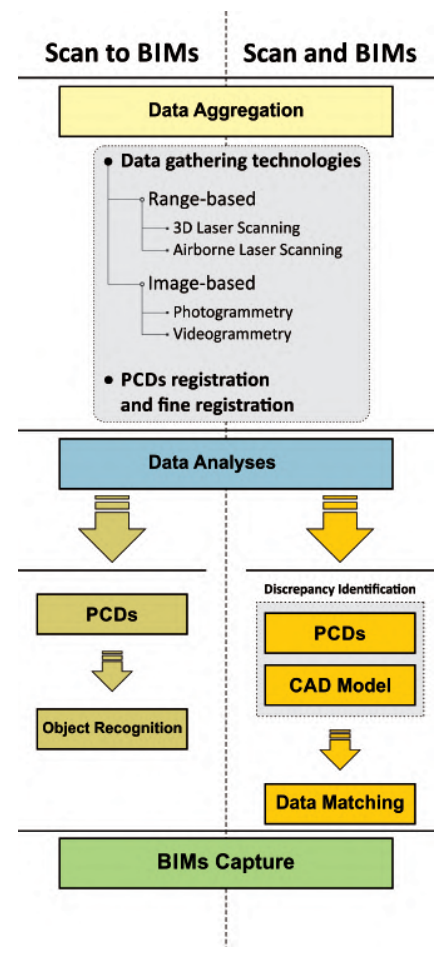

Fig. 1. The general workflow of 'Scan and BIMs' \& 'Scan to BIMs'. 
CAD model, the process of capturing BIMs is performed through 'Scan and BIMs' procedure [11]. On the other hand, in unique circumstances, such as in existing buildings and historical environments, in which 2D documents are the only available data, BIMs are mapped adopting 'Scan to BIMs' approach [12].

Historic Building Information Modelling (HBIM) has lately achieved significant attention in developing a suitable BIM framework concerning the modelling of historical monuments. The quality of the represented model, its geometric reliability [1] and, more significantly, the required asset information embedded in the generated models play a key role in elaborating a suitable HBIM framework.

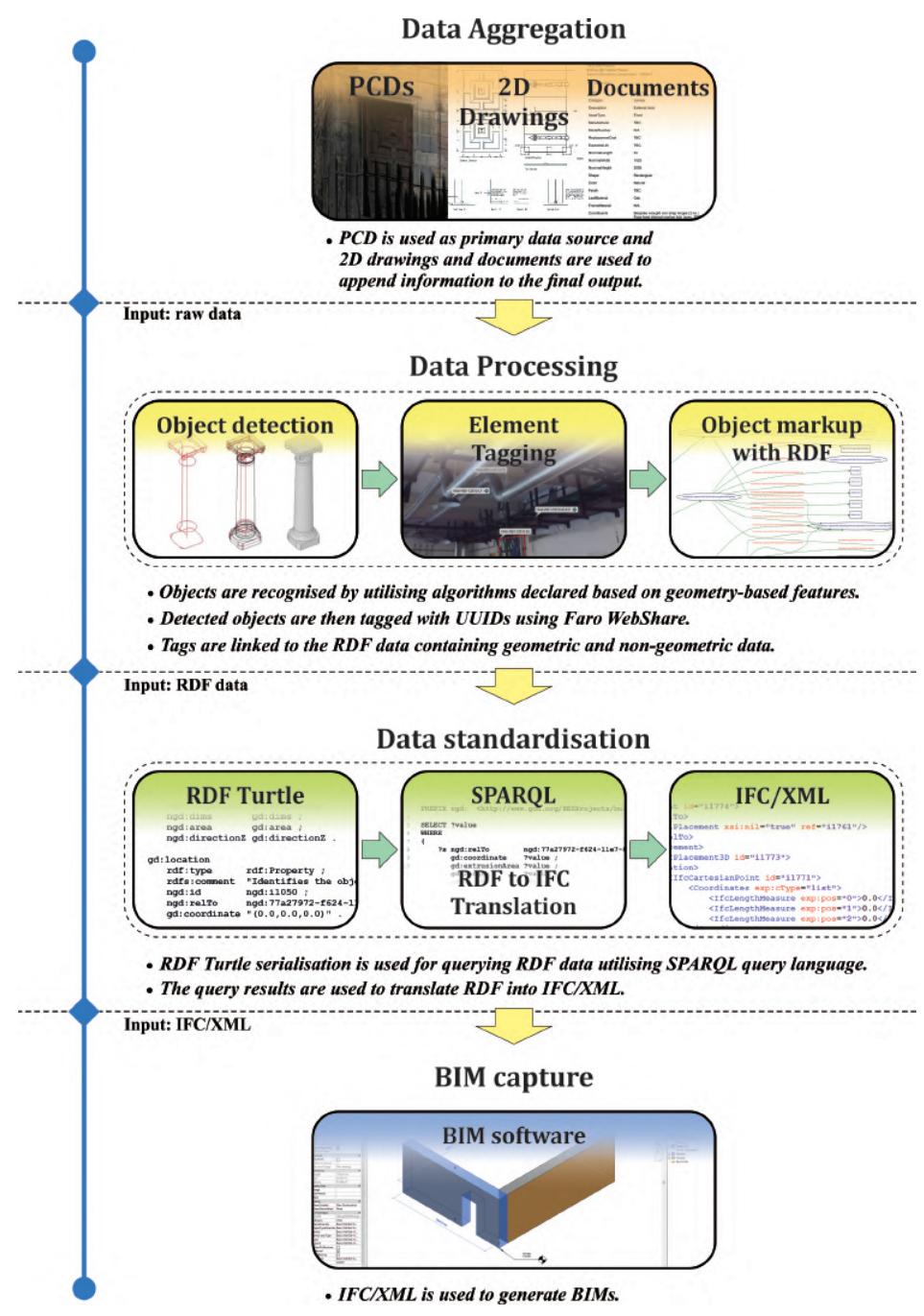

Fig. 2. The workflow of proposed approach. 
A detailed and semantic representation of HBIM can be useful to address an appropriate LoD in advancing the HBIM framework. While the use of BIM in new construction projects has lately gained significant momentum, its use in existing assets, specifically historic buildings, has been hampered by challenges surrounding the limitations of existing technologies for capturing retrofit models $[2,13]$. A variety of different technologies are available for gathering the data from an existing asset in the form of images or point measurements, including imagebased and range-based methods. Photogrammetry and Videogrammetry are the most commonly adopted methods in the image-based domain $[4,14,15,16]$. 3D laser scanning technology as a range-based method is an accurate, popular, and commonly used method for extracting the data from an existing asset in the form of Point Cloud Data (PCD) $[2,4,14]$. The challenging part of generating parametric models is to record and analyse the information included in PCDs. Although some progress has been recently made in generating non-parametric models from PCDs, a proper full-blown parametric model is still some way away.

This paper reviews the state-of-the-art to address the challenges involved in generating parametric models and managing the large-scale information (metadata) embedded in models before proposing our approach. A Semantic Webbased approach is proposed in this paper to generate parametric models and to manage the required asset information. The workflow of this ongoing project can be classified into four general steps consisting of 1) Data Aggregation, 2) Data Processing, 3) Data Standardization, and 4) BIM Capture (Fig.2). PCD extracted from 3D laser scanner is used as the primary input data along with corresponding $2 \mathrm{D}$ drawings and related documents to improve the final results. In the data processing step, building elements are recognized in PCD, and the concept of RDF is then used to mark up detected objects. The challenging part of this approach is the data standardization process where the RDF data as a Semantic Web key technology needs to be translated to Industry Foundation Classes (IFC). The final step is to import IFC as an open source data model into any BIM software that supports IFC schema.

\section{Methodology}

The research gap and the challenges involved in capturing semantic-rich threedimensional parametric models are identified by reviewing academic journals, conference proceedings, books, and applied application (e.g. reports, buildingSMART, openBIM, RDF core and APIs, etc.) that contribute to the implementation of BIM and HBIM for new buildings and retrofit assets. The focus of this review is on three key subjects, including 1) BIM process and its use in the construction industry along with associated applications used in new and existing buildings, 2) construction-related technologies, such as remote sensing (e.g. 3D laser scanning), 3) The use of Semantic Web standards, technologies, and applications in construction and other domains (e.g. RDF, SPARQL, etc.). A keyword search approach is applied to limit the scope of resources. The main keywords that are used are 'Building Information Modelling (BIM)', 'Historic Building 
Information Modelling (HBIM)', 'Parametric Modelling', 'Semantic Web', 'Resource Description Framework or Format (RDF)', SPARQL Protocol And RDF Query Language (SPARQL), and 'Industry Foundation Classes (IFC)'. The Semantic Web-related technologies are investigated to identify an appropriate architecture for marking up the detected elements and managing the large-scale information embedded in parametric models. The 'Apache Jena API' (for generating RDF graphs), 'W3C SPARQL' query language, 'IFC Open Tool' (for generating IFC), and Autodesk Revit 2016 (BIM modeller) are utilized to implement the data standardization and BIM capture processes.

\section{Related work}

In the past years or so, varying studies have been carried out to develop automated or semi-automated approaches for capturing parametric models, utilizing PCDs as the main data source. The proposed approaches can be classified into two general classes consisting of 'Scan and BIMs' and 'Scan to BIMs' (Fig.1). A variety of different data collection technologies and methods are available in the construction industry for capturing the data from a new built projects or existing assets. However, 3D laser scanning (range-based) is the most popular and adopted technology among other methods owing to the data accuracy and geometric information sufficiency $[2,10,14,15]$. The process of generating BIMs, in either case, comprises several common steps and the difference between developed approaches relies on the availability of the CAD model. In new building projects, if the CAD model is accessible, the scanned data (PCD) is matched and compared to the CAD model to identify the discrepancies between existing data sets for generating BIMs (Scan and BIMs). On the other hand, if the asset does not have an existing model, BIMs are captured by identifying elements inside the PCD (Scan to BIMs). Several approaches have been proposed in the literature to generate parametric models using the geometric features embedded in PCD, such as lines, boundaries, 2D \& 3D primitives, and so forth. Moreover, approaches proposed in the literature are supported by various declared algorithms to assist the process and to move from manually generating BIMs, which is timeconsuming, tedious and error-prone due to the human intervention $[5,17,18]$, towards an automated or semi-automated practice. Most of the approaches are declared based on the geometric features that are embedded in PCDs. In Gao et al. [11] a variety of different algorithms have been declared to detect the geometric relationship between elements in PCD and the corresponding CAD model in a real-world mechanical project. In this approach, geometry-related features are used to identify geometric primitives and to project the as-is (as-built) condition. The datasets, PCD and the available CAD model, are then matched together to identify the discrepancies, such as shape, location, dimension, and composition, between the as-designed model and as-is condition for capturing accurate as-built models. Other geometric specifications, such as region-based, primitivebased, and distribution-based characteristics, are employed in similar proposed methods for generating accurate BIMs $[6,11,19]$. 


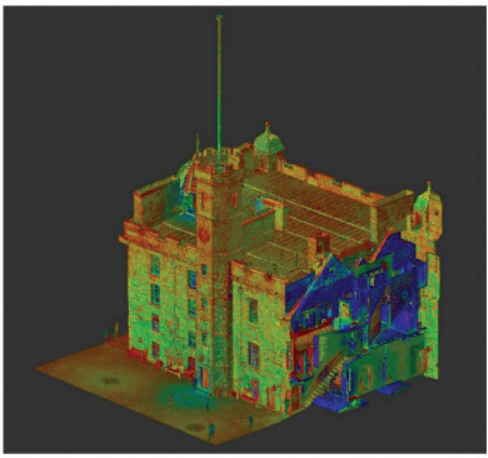

Point Cloud Data (PCD)

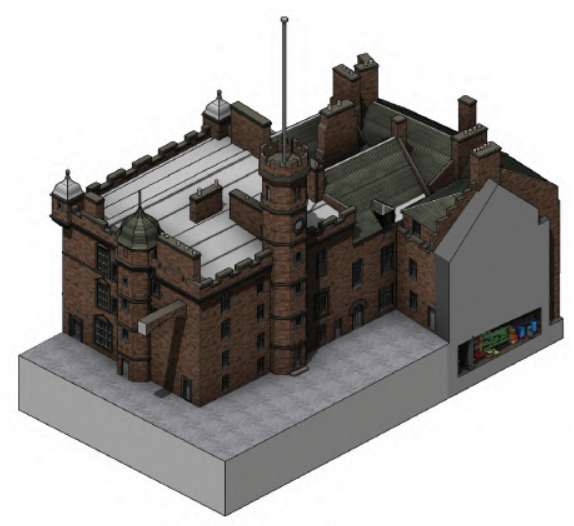

Generated model from PCD

Fig. 3. Edinburgh Castle model generated from PCDs (source: Historic Environment Scotland, 2016).

On the other hand, in some unique circumstances, particularly historical environments, the existing asset may not have a 3D CAD model, and the only available information includes $2 \mathrm{D}$ drawings and related documents. In this case, the retrofit models need to be generated from the data collected from the building (typically PCD captured by 3D laser scanner). Most approaches that are developed based on this method (Scan to BIMs) use PCD as the main data source. The approach proposed in Zhang et al. [12] focuses on detecting and extracting the planar patches of an existing asset. In this method different aspects of geometric features, like the relationship between points and linear characteristics, are used to recognize the boundaries and surfaces (geometric primitives) of 3D shapes. A similar approach is proposed in Xiong et al. [17] to capture interior building elements, such as walls, floors, ceilings, windows, and openings. The spatial-related characteristics like the connectivity, relative distance, and the contextual relationship between point elements and corresponding building components, is used to identify and extract planar patches. The detected surfaces are then interconnected to form 3D geometric primitives that are clustered in different categories (wall, floor, door, etc.) afterwards. Other similar approaches, such as Budroni A. and Boehm J. [20,21], Adan A. and Huber D. [22,23], and Okorn et al. [24] have also been developed based on the geometric features for detecting geometric primitives of a 3D shape and constructing building elements based on the identified shapes.

The use of BIM in historic environments has been recently increased among heritage communities concerning the generation and representation of comprehensive and intelligent 3D models. 3D parametric models in HBIM are used for different purposes, such as restoration, conservation, retrofitting, building analyses, and more importantly the facility management $[3,25,26,27]$. While different methods have been developed for capturing BIMs in new environment and retrofit assets (non-historical buildings), the development of approaches for gen- 
erating semantic-rich parametric models in heritage domain has recently gained a lot of momentum. One of the challenges involved in using BIM in historical environments is that historic buildings, compared to new build projects, include more complex components and shapes $[27,28]$ that are not supported by commercial BIM software. Moreover, commercial BIM software are generally designed to model new buildings and are limited to irregular and complex geometries that occur in HBIM [3,29]. The approach proposed in Oreni et al. [26] focuses on the restoration requirements of a historical building. In this approach different features of NURBS (Non-Uniform Rational Base-Spline) have been utilized to reconstruct geometric objects focusing on the mutual relationship between them using PCD as the main data source. The detected geometric elements are then converted into building components. A similar method is proposed in Barazzetti et al. [27] to capture parametric objects of an existing building as well as a historic bridge using NURBS characteristics. The workflow of the proposed approach starts with extracting the discontinuity lines and corresponding surfaces from PCDs using the NURBS attributes, and the geometric shapes are generated by interconnecting the extracted planar patches.

Despite mathematic-based methods, there are other approaches proposed in the literature that focus on developing libraries of building elements for capturing BIMs in HBIM domain [30]. In Dore \& Murphy [3] a semi-automated approach is developed that is based on a library of different building elements supported by a rule-based algorithm. In this method, building elements, extracted from the range-based (PCD) and image-based (images) technologies, are matched to the components that are created and stored in a pre-defined library for capturing retrofit BIMs. More information regarding the creation of building elements library can be found in Murphy et al. [30]. In addition, there are other proposed approaches, which are developed based on element libraries and architectural ontologies, for manually or semi-automatically BIMs generation using PCD as the main data source $[1,31,32]$.

\section{Challenges and limitations}

Although some progress has been recently made in the generation of building geometries or parametric models using PCD as the main data source, a proper full-blown parametric model is still some way away. The fact is that the process of extracting appropriate details of an existing asset, including the accuracy of geometries as well as the level of automation, varies from one method to another. Hence, one single declared method cannot be used for different objects and environments sufficiently [33]. Moreover, the structure of developed approaches is based on the project requirements, such as the accuracy of models, the real representation of the model, information attached to the extracted geometries, the Level of Detail (LoD), etc. Different types of challenges are involved in extracting the information from PCDs and generating corresponding building components. The integration of BIM software and data collection technologies is the most common limitation [34], particularly in historical environments owing to the 


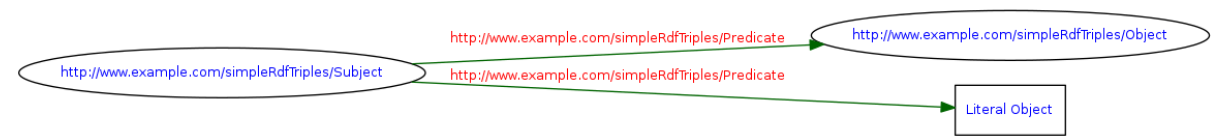

Fig. 4. RDF statement (triple).

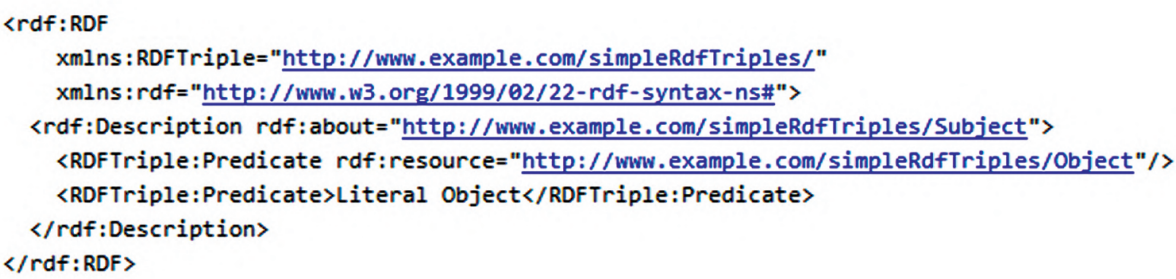

Fig. 5. RDF/XML format for the RDF graph in Fig.4.

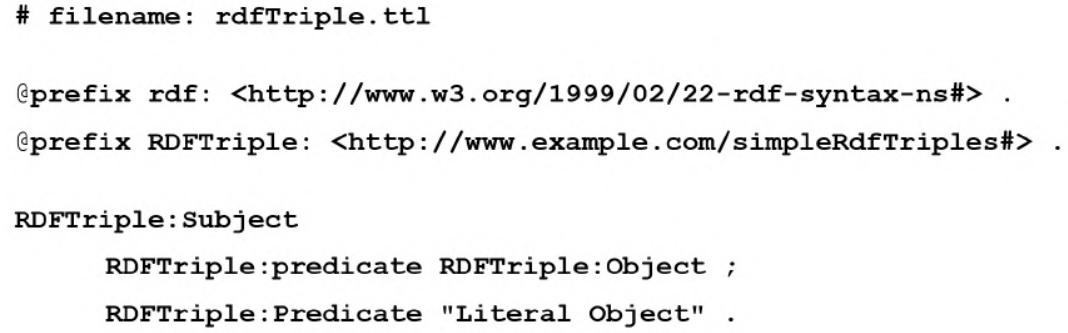

Fig. 6. RDF Turtle format for the RDF graph in Fig.4.

complexity and unique form of each building component. New build projects contain regular shapes and components while historical buildings contain building elements with irregular shapes that remain as simple geometry primitives during the process of capturing BIMs due to the limitation of commercial BIM software. The level of required information embedded in extracted models, which is an important part of Building Information Modelling process, is another challenge involved in BIM capture. Furthermore, the process of attaching required asset information to the captured elements is the challenging part of generating semantic-rich parametric models in HBIM, and this part is currently carried out manually. The Edinburgh Castle BIM project (Fig.3), as a case study in this ongoing research, carried out by Historic Environment Scotland (HES) could be a good example for mapping BIMs from PCD manually. The management and manipulation of large-scale information embedded in extracted elements and BIM models during the asset lifespan is another challenge that needs to be addressed for developing a suitable BIM framework for retrofit assets and historical buildings. 


\section{Semantic Web Technologies}

Semantic Web (SW) as a set of technologies and standards provides a suitable framework for the store, share, and reuse of the information [35] on the web. In the past years or so, the use of SW technologies has gained growing levels of popularity and interest in different domains, including the AEC. In McGibbney \& Kumar [36] the SW technology is used for creating a framework (model) for the legislation in AEC domain. Another example of the SW use could be the case study carried out by Hernandez et al. [37,38] in cultural heritage domain. Resource Description Frame-work or Format (RDF), as a structure on the web, is a commonly used SW technology (also known as SW standard format) for describing and interchanging the information in the form of metadata [39]. RDF is structured based on a simple architecture, and the basic logic of RDF makes it capable to manage and process large-scale data, and it also supports a variety of different contexts [40]. The representation of RDF is based on simple statements also known as triples. An RDF statement consists of a subject (instance), predicate (property), and an object (value). RDF graph is constructed by matching the subject to the object through the predicate (Fig.4). The subject and predicate are declared as URIs, and the object can be declared either as a URI or literal value (string value) [41,42]. The concept of RDF supports varying formats, such as RDF XML, RDF Turtle, etc. The RDF XML format for the same RDF graph illustrated in Figure 4 is shown in Figure 5, and the Turtle version for the same graph is shown in Figure 6. The RDF graph data compared to other graph databases (DBs), such as Relational DBs and Hierarchical DBs, does not have a concept of root or hierarchy which enables RDF to relate resources to another without any hierarchical relationships between them [40].

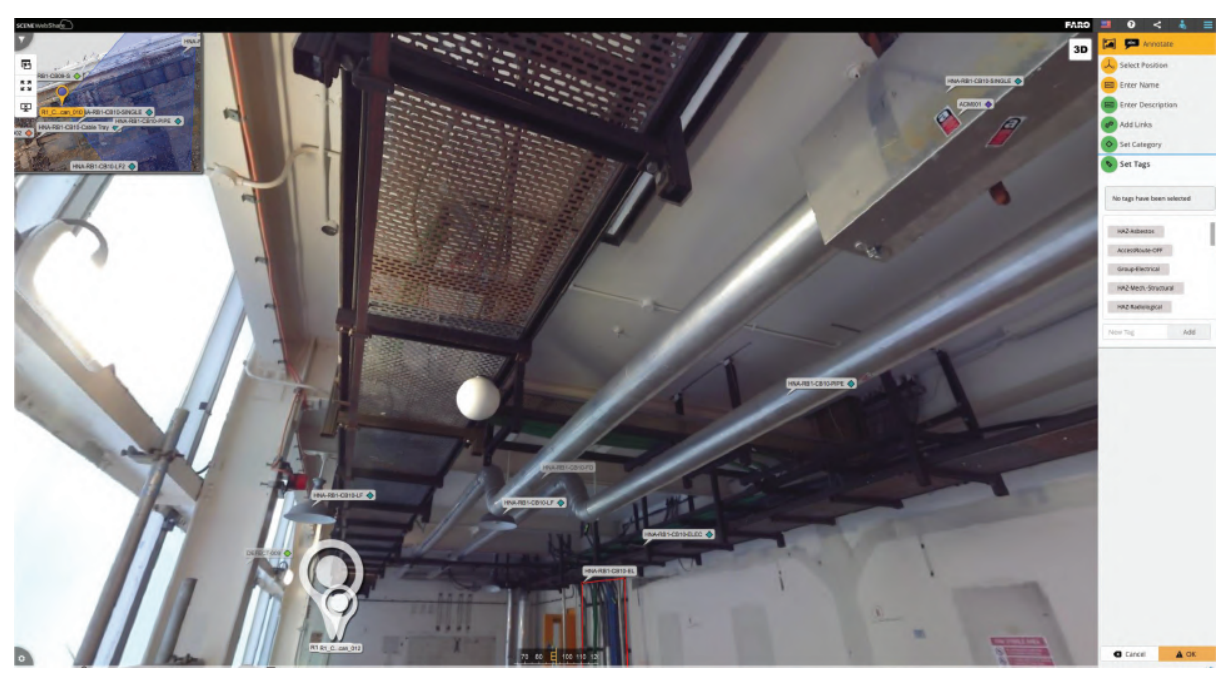

Fig. 7. Tagging building elements in Faro WebShare [44]. 


\section{Parametric modeling}

The general workflow of the proposed approach, as described in Figure 2, consists of four steps starting with the data acquisition. PCD is the main source of data in this project, and other related data, like 2D drawings and corresponding documents, are used in addition to the PCD to facilitate the final result. The second step is to process the collected data and use the concept of RDF to mark up elements identified in PCD. The principal focus of this project is the data standardization process as the challenging part of this ongoing research where RDF data as a serialization needs to be translated into IFC format as a data model. The final step of the proposed method is to use the IFC to generate the BIM model by importing the IFC data model into BIM software that support IFC.

\subsection{RDF markup \& Data standardization}

The first step in data processing section is the object recognition process. Building elements are recognized using the geometric feature in PCD. As mentioned in Section 3, several approaches have been developed for detecting building object in PCD, but with a varying of success. The elements that are detected in PCD are geometric primitives that do not contain required asset information needed to

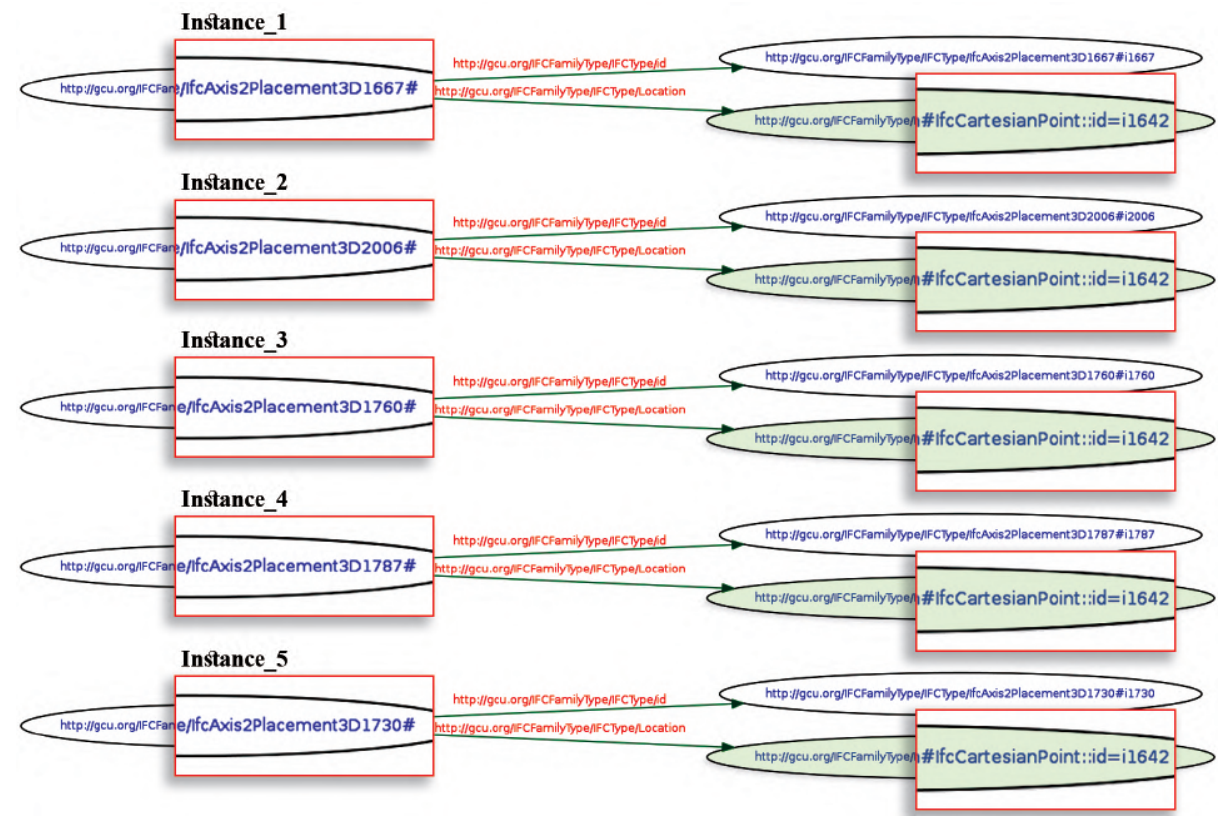

Fig. 8. RDF sub-graphs for five different instances (subjects) that contain same value for the location property. 
facilitate the BIM process and the future management of an asset. In this project, we use the concept of RDF to mark up identified elements and to append required asset information to building objects. The recognized building elements are first tagged by a GUID (Globally Unique IDentifier) or UUID (Universally Unique IDentifier) which is then linked with the corresponding RDF graph or any other RDF serializations, like .ttl, .xml, .json, etc. There are different commercial software available that can be used for tagging elements (assigning the GUID to elements) directly in PCD, such as FARO WebShare (Fig.7). The use of RDF provides the fundamentals for the analysis, management, and interoperability of large-scale information [43]. The interoperability feature of RDF is used for sharing the information throughout the process efficiently. In a building, including new, retrofit, and historical assets, different detected elements might share similar information, and in current practice, the data is attached to the element individually. However, RDF provides the opportunity to merge similar information and exchange semantic information [40] that can be used among different building components. The sharing characteristic of RDF accordingly reduces the size of the information. The associated information for an element is firstly declared in separate RDF sub-graphs (Fig.8), and the main RDF graph is generated by merging sub-graphs. In Figure 9, an RDF graph (merged graph model) for a wall demonstrates an IfcCartesianPoint with a unique id (i1642) that is a shared value for five different instances (sub-graphs that are declared separately) and an instance for the next corresponding values. Instead of declaring the value for each instance separately, RDF automatically identifies and merges similar values and represents an individual value for different subjects (in this case, five instances) which reduce the scale of the information accordingly.

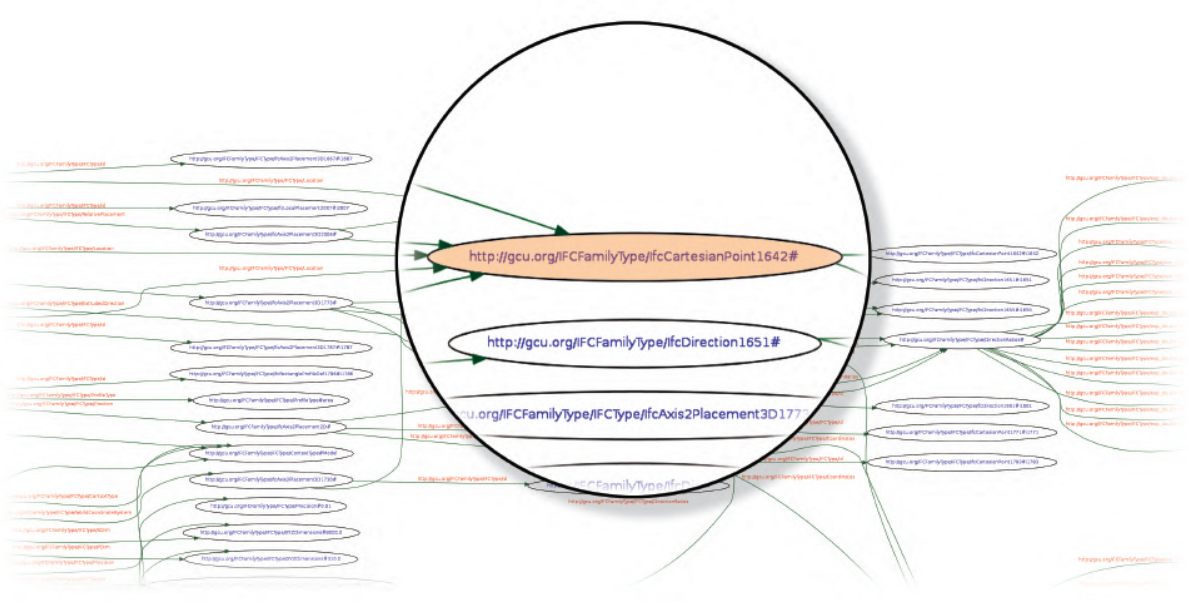

Fig. 9. Shared value for five instances in RDF graph generated by merging sub-graphs. 


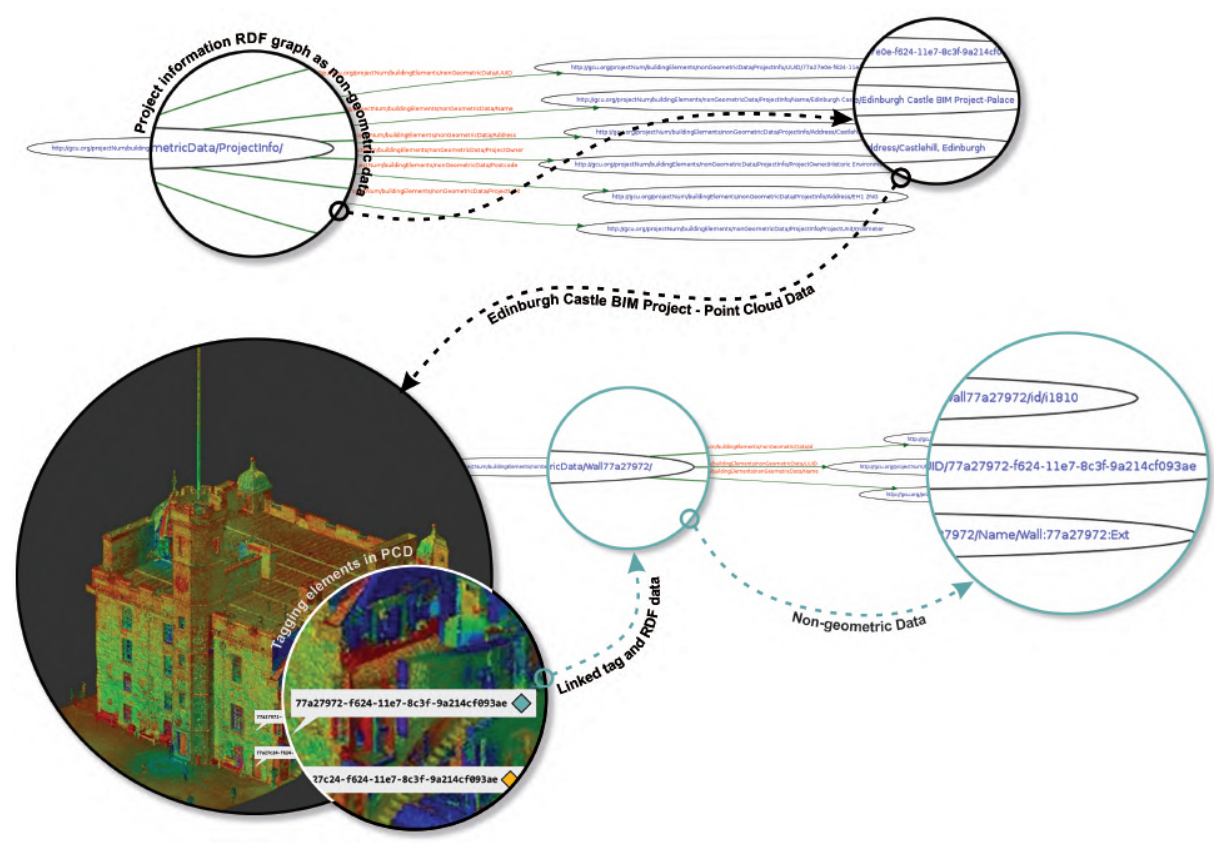

Fig. 10. Marking up building elements using the concept of RDF.

A parametric model contains two types of information, consisting of geometric information (e.g. location, dimension, area, element relationship, etc.) and non-geometric information that cannot be extracted from PCD (e.g. name, GUID, material, and other required asset information). The concept of RDF offers the opportunity to manage different information with varying vocabularies. The information in the form of RDF serializations linked with the tags in PCD (Fig.10) is stored, shared on the web along with the corresponding model as the linked data. In other words, if the information for a building element changes in the model or in RDF data during the life-cycle of an asset, the alterations will be applied to the project accordingly. The other advantage of using RDF for managing the information is that stored data can be reused for other building components in different projects. In general terms, the use of SW technologies enhances and facilitates the access to the data archives, the data navigation process, metadata representation with multiple formats, the integration between multiple data sources with different vocabularies and structures, etc.

The challenging part of this ongoing research is the data standardization process. In this step, primary RDF data as a serialization, which is a set of byte streams stored in a file or memory and can be reconstructed and reused later if required, needs to be translated to IFC as a data model and the common and globally used standard data format within the construction industry for the information integration and exchange [2,5].The first step of this process is to extract the information from RDF data that is used to mark up elements in 


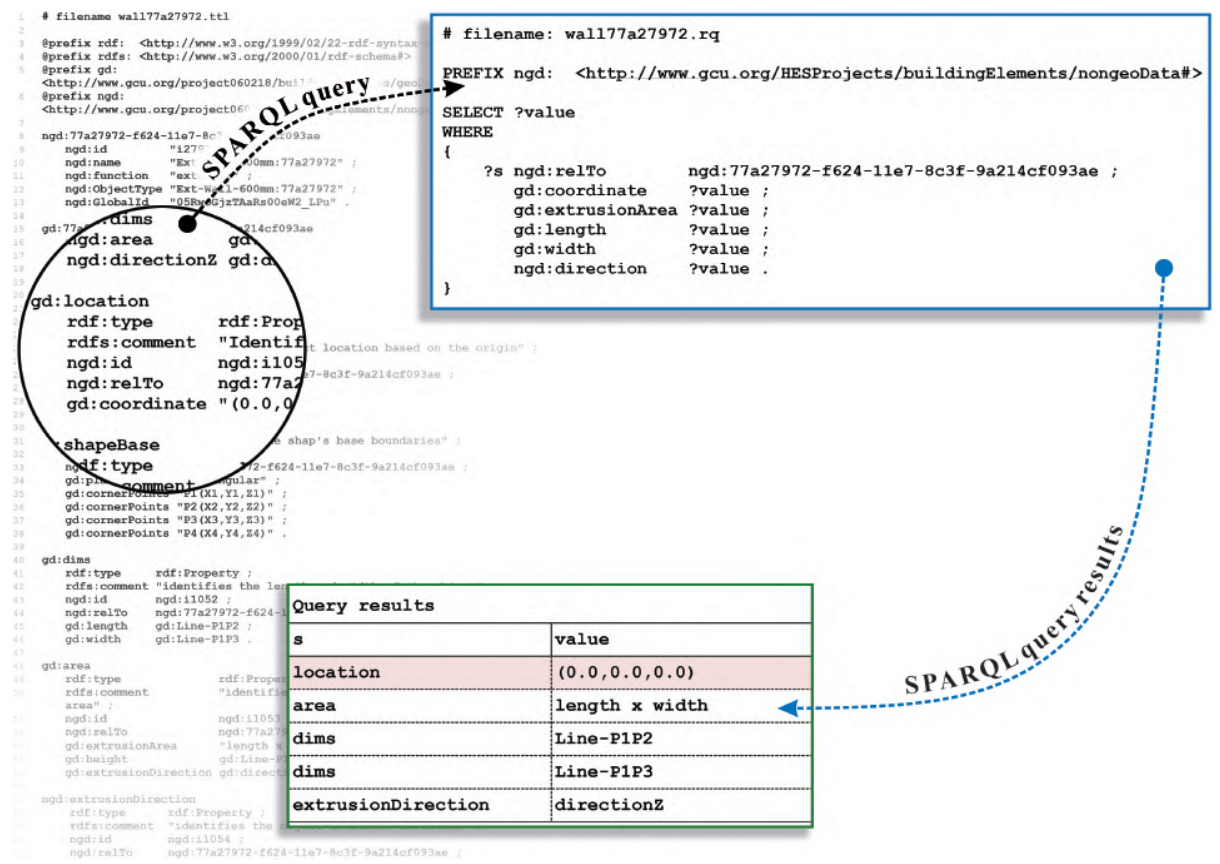

Fig. 11. Extracting the information from RDF data (RDF Turtle format) using SPARQL query language.

PCD by linking RDF to the tags. We use SPARQL (Sparql Protocol And RDF Query Language) - as the standard query language designed for Semantic Web to query and pull data from RDF. SPARQL is not limited to the RDF data and it can be also applied to other data serialisation $[45,46]$. One of the advantages of SPARQL is that it can be applied to either a single RDF statement or to an RDF graph that is structured by merged sub-graphs. In this project, the Turtle format of RDF is used to extract required information for the translation process. The SPARQL query results - information extracted from RDF data are then used to generate corresponding entities for IFC XML. For example, The RDF statement for a wall consists of an UUID (the tag of the wall in PCD linked to the corresponding RDF data as the subject represented by an URI), location as the property of the wall (predicate represented by an URI), and the value of the property (object represented as a literal or URI). The SPARQL query language is then used to extract the data associated with the wall by querying for the location value (object) which is a point coordinate(x,y,z) (Fig.11). The query results (data extracted form RDF Turtle) are then used to implement the RDF to IFC translation process. The IFC XML ObjectPlacement entity as a sub-entity of IfcWall or IfcWallStandardCase is then used to locate the wall in project according to the project origin point (Fig.12). Depending on the structure of the wall, IfcWall or IfcWallStandardCase entity is used in IFC to represent 


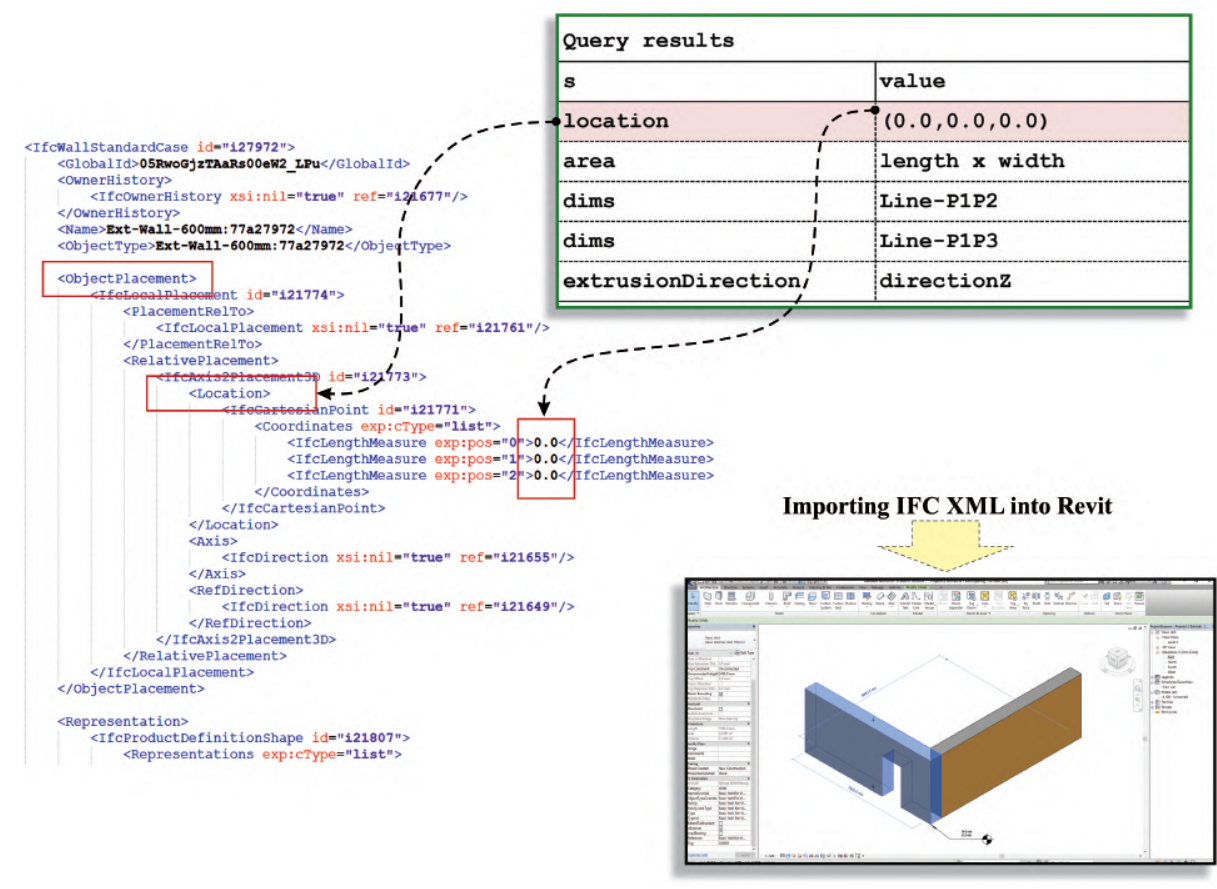

Fig. 12. Using SPARQL query results to translate RDF data to IFC XML format, and creating the IFC XML to generate the parametric model in Autodesk Revit BIM software.

a wall, including geometric and non-geometric data. The non-geometric data, such as Name, ObjectType, identifier (id), and GlobalId as sub-entities for the wall entity, are accordingly appended to the corresponding element. The same process can be applied to other entities, such as the wall layouts and materials, and other elements like doors and windows and corresponding openings. The final step is to import the generated IFC XML into any BIM software that supports IFC format to map parametric models.

\section{Conclusion}

The aim of the proposed approach in this paper is to address challenges and limitations involved in generating parametric models for retrofit assets, particularly historical buildings, and to provide a suitable BIM framework for mapping BIMs. Several approaches have been recently developed to detect and model building components using PCD as the main data source. However, a proper parametric model is still some way away. The approach presented in this paper uses the concept of Semantic Web technologies to provide an effective and efficient solution to address identified challenges. The use of RDF as a Semantic Web technology 
makes the proposed method capable of managing the large-scale information involved in the parametric modelling process. RDF also gives the opportunity to produce large-scale databases of identified objects, that are labelled throughout the process, using simple RDF statements, and to share the stored information with the same components identified in one project and similar objects in other projects. Several approaches have been structured based on object libraries, and this advantage of Semantic Web technologies can be a proper solution for dealing with metadata.

With regard to retrofit assets, building models change during the life-cycle of an asset owing to the alterations produced during the remodeling process, and consequently the corresponding information change. In our approach, we use the concept of RDF to provide a suitable solution for simplifying and manipulating the information during applied changes. The third step of the proposed approach, data standardization, is the core and challenging part of this ongoing research where RDF as a Semantic Web technology and a serialization needs to be translated to IFC as an open source data model. Currently, some steps of this research are carried out manually, and the next step of this work is to change the workflow of the proposed method to an automated process. The primary results of this research are quite promising and should be of interest to the modelling of all kinds of building components, particularly historical buildings.

\section{Acknowledgement}

Authors would like to express their gratitude to Dr Vajira Premadasa of Historic Environment Scotland for providing assistance and support in the research presented in this paper.

\section{References}

1. Quattrini, R., Malinverni, E., Clini, P., Nespeca, R., Orlietti, E.: From TLS to HBIM. High quality semantically-aware 3D modeling of complex architecture. The International Archives of Photogrammetry, Remote Sensing and Spatial Information Sciences 40(5), 367-374 (2015).

2. Volk, R., Stengel J., Schultmann, F.: Building Information Modeling (BIM) for existing buildings - Literature review and future needs. Automation in construction 38, 109-127 (2014).

3. Dore, C., Murphy, M.: Historic Building Information Modelling (HBIM). Handbook of Research on Emerging Digital Tools for Architectural Surveying, Modeling, and Representation 1, 239-280 (2015).

4. Brumana, R., Oreni, D., Cuca, B., Binda, L., Condoleo, P., Triggiani, M.: Strategy for integrated surveying techniques finalized to interpretive models in a byzantine church, Mesopotam, Albania. International Journal of Architectural Heritage 8(6), 886-924 (2014).

5. Zhang, S., Teizer, J., Lee, J.-K., Eastman, C. M., Venugopal, M.: Building information modeling (BIM) and safety: Automatic safety checking of construction models and schedules. Automation in Construction 29, 183-195 (2013). 
6. Gao, T., Akinci, B., Ergan, S., Garrett, J.: An approach to combine progressively captured point clouds for BIM update. Advanced Engineering Informatics 29(4), 1001-1012 (2015).

7. Brilakis, I., Lourakis, M., Sacks, R., Savarese, S., Christodoulou, S., Teizer, J., Makhmalbaf, A.: Toward automated generation of parametric BIMs based on hybrid video and laser scanning data. Advanced Engineering Informatics 24(4), 456-465 (2010).

8. Hayne, G., Kumar, B., Hare, B.: The Development of a Framework for a Design for Safety BIM tool. In: Computing in Civil and Building Engineering (2014), pp. 49-56, Orlando (2014).

9. Turkan, Y., Bosche, F., Haas, C., T., Haas, R.: Automated progress tracking using $4 \mathrm{D}$ schedule and 3D sensing technologies. Automation in Construction 22, 414-421 (2012).

10. Tang, P., Huber, D., Akinci, B., Lipman, R., Lytle, A.: Automatic reconstruction of as-built building information models from laser-scanned point clouds: A review of related techniques. Automation in construction 19(7), 829-843 (2010).

11. Gao, T., Ergan, S., Akinci, B., Garrett, J.: Evaluation of different features for matching point clouds to building information models. Journal of Computing in Civil Engineering 30(1), 1-13 (2014).

12. Zhang, G., Vela, P., A., Karasev, P., Brilakis, I.: A Sparsity-Inducing OptimizationBased Algorithm for Planar Patches Extraction from Noisy Point-Cloud Data. Computer-Aided Civil and Infrastructure Engineering 30(2), 85-102 (2015).

13. Chevrier, C., Charbonneau, N., Grussenmeyer, P., Perrin, J., P.: Parametric documenting of built heritage: $3 \mathrm{D}$ virtual reconstruction of architectural details. International Journal of Architectural Computing 8(2), 135-150 (2010).

14. Bosche, F., Forster, A., Valero, E.: 3D SURVEYING TECHNOLOGIES AND APPLICATIONS: POINT CLOUDS AND BEYOND. Heriot-Watt University, Edinburgh (2015).

15. Bosche, F.: Automated recognition of 3D CAD model objects in laser scans and calculation of as-built dimensions for dimensional compliance control in construction. Advanced engineering informatics 24(1), 107-118 (2010).

16. Golparvar-Fard, M., Bohn, J., Teizer, J., Savarese, S., Pena-Mora, F.: Evaluation of image-based modeling and laser scanning accuracy for emerging automated performance monitoring techniques. Automation in Construction 20(8), 1143-1155 (2011).

17. Xiong, X., Adan, A., Akinci, B., Huber, D.: Automatic creation of semantically rich 3D building models from laser scanner data. Automation in Construction 31, 325-337 (2013).

18. Son, H., Kim, C.: Automatic segmentation and 3D modeling of pipelines into constituent parts from laser-scan data of the built environment. Automation in Construction 68, 203-211 (2016).

19. Huber, D., Akinci, B., Oliver, A., A., Anil, E., Okorn, B., E., Xiong, X.: Methods for automatically modeling and representing as-built building information models. In: Proceedings of the NSF CMMI Research Innovation Conference, Atlanta (2011).

20. Budroni, A., Bohm, J.: Automated 3D reconstruction of interiors from point clouds. International Journal of Architectural Computing 8(1), 55-73 (2010a).

21. Budroni, A., Bohm, J.: Automatic 3D modelling of indoor manhattan-world scenes from laser data. In: Proceedings of the International Archives of Photogrammetry, Remote Sensing and Spatial Information Sciences, 115-120 (2010b). 
22. Adan, A., Huber, D.: Reconstruction of wall surfaces under occlusion and clutter in 3D indoor environments. Robotics Institute, Carnegie Mellon University, Pittsburgh, PA CMU-RI-TR-10-12, (2010).

23. Adan, A., Huber, D.: 3D reconstruction of interior wall surfaces under occlusion and clutter. In 3D Imaging, Modeling. In: 2011 International Conference on Processing, Visualization and Transmission (3DIMPVT), IEEE, 275-281 (2011).

24. Okorn, B., Xiong, X., Akinci, B., Huber, D.: Toward automated modeling of floor plans. In: Proceedings of the symposium on 3D data processing, visualization and transmission 2, (2010).

25. Oreni, D., Brumana, R., Georgopoulos, A., Cuca, B.: HBIM for conservation and management of built heritage: Towards a library of vaults and wooden bean floors. ISPRS Annals of Photogrammetry, Remote Sensing and Spatial Information Sciences 5, W1 (2013).

26. Oreni, D., Brumana, R., Della Torre, S., Banfi, F., Previtali, M.: Survey turned into HBIM: the restoration and the work involved concerning the Basilica di Collemaggio after the earthquake (L'Aquila). ISPRS Annals of the Photogrammetry, Remote Sensing and Spatial Information Sciences 2(5), 267-273 (2014).

27. Barazzetti, L., Banfi, F., Brumana, R., Previtali, M., Roncoroni, F.: BIM from laser scans not just for buildings: NURBS-based parametric modeling of a medieval bridge. ISPRS Annals of Photogrammetry, Remote Sensing \& Spatial Information Sciences 3(5), 51-56 (2016).

28. Barazzetti, L., Banfi, F., Brumana, R., Gusmeroli, G., Oreni, D., Previtali, M., Roncoroni, F., Schiantarelli, G.: BIM from laser clouds and finite element analysis: combining structural analysis and geometric complexity. The International Archives of Photogrammetry, Remote Sensing and Spatial Information Sciences 40(5), 345350 (2015).

29. Murphy, M., McGovern, E., Pavia, S.: Historic building information modelling (HBIM). Structural Survey, 27(4), 311-327 (2009).

30. Murphy, M., McGovern, E. and Pavia, S.: Historic Building Information ModellingAdding intelligence to laser and image based surveys of European classical architecture. ISPRS journal of photogrammetry and remote sensing, 76, 89-102 (2013).

31. Attar, R., Prabhu, V., Glueck, M., Khan, A.: 210 King Street: a dataset for integrated performance assessment. In: Proceedings of the 2010 Spring Simulation Multiconference, Society for Computer Simulation International, 177-180 (2010).

32. Chevrier, C., Perrin, J., P.: Generation of architectural parametric components. In: CAAD futures 09, Les Presses de l'Universit de Montral, 105-118 (2009).

33. Fassi, F., Achille, C., Fregonese, L.: Surveying and modelling the main spire of Milan Cathedral using multiple data sources. The Photogrammetric Record 26(136), 462-487 (2011).

34. Apollonio, F., I., Gaiani, M., Sun, Z.: BIM-based modeling and data enrichment of classical architectural buildings. SCIRES-IT-SCIentific RESearch and Information Technology, 2(2), 41-62 (2012).

35. Yu, L.: A developers guide to the semantic Web. Springer Science \& Business Media, Berlin, (2011).

36. McGibbney, L., J., Kumar, B.: A Framework for Regulatory Ontology Construction within AEC Domain. In: Ontology in the AEC Industry, 193-215 (2015).

37. Hernndez, F., Rodrigo, L., Contreras, J., Carbone, F.: Building a cultural heritage ontology for Cantabria. In: Annual Conference of CIDOC, 1-14 (2008). 
38. Carrascal, F., H., Rodrigo, L., Contreras, J., Carbone, F.: Cantabria cultural heritage semantic portal. In: Proceedings of the 2007 International Conference on Semantic Web Challenge 295, 9-16 (2007).

39. Domingue, J., Fensel, D., Hendler, J., A.: Handbook of semantic web technologies. Springer Science \& Business Media, Berlin, (2011).

40. Powers, S.: Practical RDF: solving problems with the resource description framework. Sebastopol, CA: O'Reilly Media, Inc., (2003).

41. Cyganiak, R., Wood, D., Lanthaler, M.: W3C Recommendation Homepage, https://www.w3.org/TR/rdf11-concepts/, last accessed 2017/09/10.

42. Klyne, G., Carroll, J., J.: W3C Recommendation Homepage, https://www.w3.org/TR/2004/REC-rdf-concepts-20040210/\#section-Concepts, last accessed 2017/09/10.

43. Antoniou, G., Van Harmelen, F.: A semantic web primer. 2nd Edition. MIT press, (2008).

44. Dolphin, J.: The Application of Building Information Modelling to the UK Nuclear Decommissioning Industry. MSc Dissertation, Glasgow Caledonian University, Glasgow, (2016).

45. DuCharme, B.: Learning SPARQL: querying and updating with SPARQL 1.1. O'Reilly Media, Inc., (2013).

46. Cure, B., Blin, G.: RDF database systems: triples storage and SPARQL query processing. Morgan Kaufmann, (2014). 